EPJ Web of Conferences 106, 01009 (2016)

DOI: $10.1051 /$ epjconf/201610601009

(C) Owned by the authors, published by EDP Sciences, 2016

\title{
Local Neutron Flux Distribution Measurements by Wire-Dosimetry in the AMMON Experimental Program in the EOLE Reactor
}

\author{
A. Gruel ${ }^{1, a}$, J. Di Salvo ${ }^{1}$, A. Roche ${ }^{1}$, J.-M. Girard ${ }^{1}$, H. Philibert ${ }^{1}$, J. Bonora ${ }^{1}$, J.-F. Ledoux ${ }^{1}$, C. Morel $^{1}$, \\ A. Lecluze ${ }^{1}$, A. Foucras ${ }^{1}$, C. Vaglio-Gaudard ${ }^{2}$, and A.-C. Colombier ${ }^{2}$ \\ ${ }^{1}$ CEA, DEN/DER/SPEx, Cadarache, 13108 St. Paul lez Durance, France \\ ${ }^{2}$ CEA, DEN/DER/SPRC, Cadarache, 13108 St. Paul lez Durance, France
}

\begin{abstract}
Dosimetry measurements were carried out during the AMMON experimental program, in the EOLE facility. Al-0.1 wt $\%$ Au wires were positioned along curved fuel plates of JHR-type assemblies to investigate the azimuthal and axial gold capture rate profiles, directly linked to the thermal and epithermal flux. After irradiation, wires were cut into small segments (a few $\mathrm{mm}$ ), and the gold capture rate of each part was measured by gamma spectrometry on the MADERE platform. This paper presents results in the "hafnium" configuration, and more specifically the azimuthal flux profile characterization. The final uncertainty on each measured wire lies below 1\% (at 2 standard deviations). Experimental profiles are in a good agreement against Monte Carlo calculations, and the $4 \%$ capture rate increase at the plate edge is well observed. The flux dissymmetry due to assembly position in the core is also measured, and shows a 10\% discrepancy between the two edges of the plate.
\end{abstract}

\section{Introduction}

Coinducted between 2010 and 2013, the AMMON experimental program [1] in the French EOLE Zero Power Reactor located in Cadarache [2], was devoted to the experimental validation of the HORUS calculation scheme, dedicated to the future Jules Horowitz Material Testing Reactor (JHR) [3]. The goal of this program was to determine the biases and uncertainties associated with the calculation of the main JHR neutron safety parameters (reactivity, absorber reactivity worth, power distribution, and kintics parameters) and performance parameters (such as fast flux in the core).

In particular, dosimetry measurements carried out during this experimental program concerned:

- spectral indexes measurements, captured with small activation foils of various materials (gold, nickel, cobalt, etc.), directly inserted in the assemblies centers or inside an aluminum overclad used in place of fuels pins in the buffer zone;

- power monitoring;

- flux distribution measurements with foils or aluminum gold alloy wires (Al-Au).

\footnotetext{
${ }^{\text {a }}$ Corresponding author: adrien.gruel@cea.fr
}

This is an Open Access article distributed under the terms of the Creative Commons Attribution License 2.0, which permits unrestricted use, distribution, and reproduction in any medium, provided the original work is properly cited. 
This paper focuses on the flux measurements performed in the experimental zone, especially between the curved plates of the JHR fuel assemblies. These measurements are complementary to gamma spectrometry measurements of the fission products decay, to determine the azimuthal [4] or axial power distribution in the plates. Results concerning axial dosimetry measurements can be found in Ref. [5].

The goal of the azimuthal measurements was to quantify the local flux increase at the plate edges or close to the aluminum stiffeners. A second effect caused by the flux distortion linked to the assembly position in the core has been studied. Experimental versus computed profiles, for several plates measured on two positions of the dismountable assembly in the AMMON core are presented.

\section{The AMMON Experimental Program}

\subsection{Experimental Mock-up}

The AMMON core consists of a central hexagonal aluminum box, with 7 JHR fuel assemblies, surrounded by a buffer zone composed of standard PWR- $\mathrm{UO}_{2}$ fuel pins. Each assembly is made of 24 curved plates, divided in three $120^{\circ}$-sectors separated by aluminum stiffeners. Plates are usually numbered by increasing order from center to periphery, from 1 to 8 (Fig. 1). One assembly can be fully disassembled to enable local fission rates measurements in the plates by gamma spectrometry or to place wire dosimeters along the plates.

In the "hafnium" configuration, a hafnium rod was inserted in the center of the central assembly in order to qualify the neutronic and photonic effects of the future JHR control rods. During this phase, azimuthal dosimetry measurements were performed to assess the gold capture rate profile, i.e. the thermal and epithermal flux profile, in the water channels between the plates (Fig. 2, water channels highlighted in white).

In the central assembly, capture rates were measured on plates 4 (water channel between plates 4 and 5) and 7 (water channel between plates 7 and 8) of sector I, which is a sector in a nominal configuration, and on the plates 4 and 7 of sector III, perturbed by the nearby assembly stiffener (Fig. 2(a)). On a peripheral assembly, the capture rate of plate 7 in sector I was measured (Fig. 2(b)).

\subsection{Dosimetry Measurements}

Instrumentation introduced between two plates had to be as non-intrusive as possible, as the distance between two plates is less than $2 \mathrm{~mm}$. Using an extended detector that can be split into small parts after irradiation, to reconstruct the neutron spatial flux shape has many advantages:

- a single irradiation is required to determine axial or azimuthal gradient, obtained thanks to postirradiation measurements;

- heterogeneity introduced between two plates is substantially decreased by using a unique, transparent to neutrons, material.

For these reasons, $1 \mathrm{~mm}$-diameter wires composed of Al-0.1 wt $\%$ Au were used. The small quantity of gold in the wires induces a very small perturbation at the measurement position, and keeps sufficient count rates to reduce statistical uncertainties after irradiation. The wires were placed directly on the fuel plate, along the plate width ( 3 to $9-\mathrm{cm}$ long wires, depending on the plate size). The positioning of a wire on a fuel plate is shown Fig. 3 .

After irradiation, the wires were cut into small segments (from 2 to $5 \mathrm{~mm}$-long) and the gold capture rate was measured at the MADERE platform [6]. The wires were measured on the PASTAGA device, which consists of a carousel equipped with $4 \mathrm{NaI}$ detectors, allowing measurements of up to 51 samples. 


\section{$15^{\text {th }}$ ISRD}

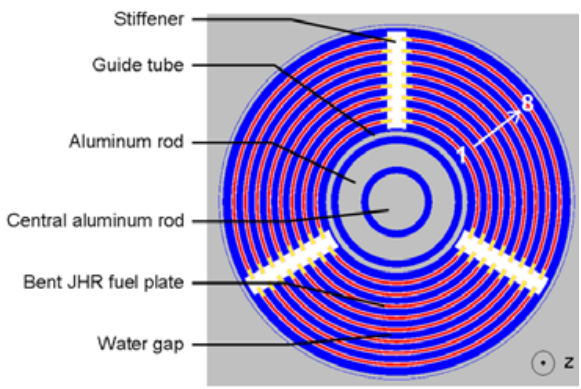

Figure 1. Radial view of a JHR fuel assembly.

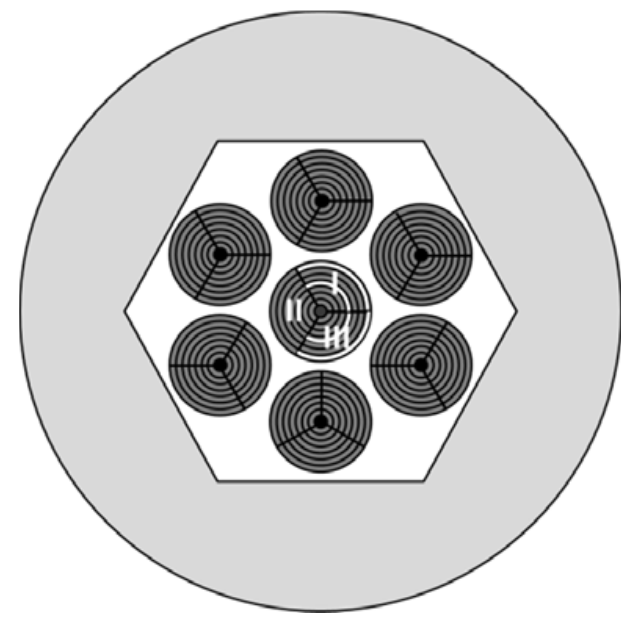

(a)

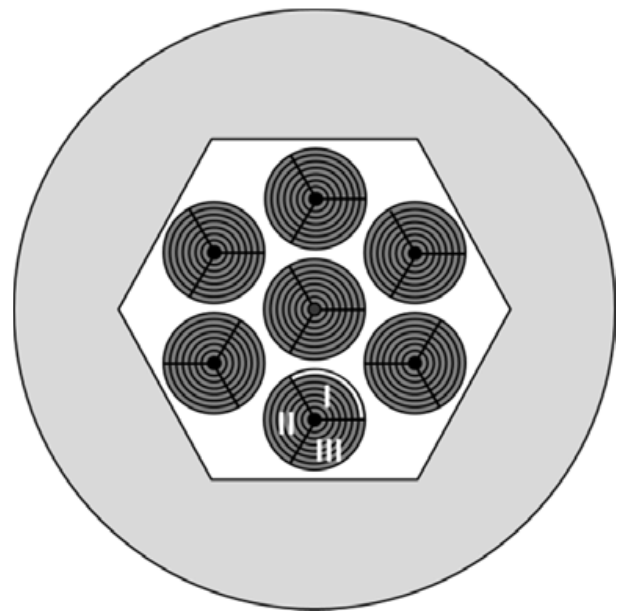

(b)

Figure 2. Schematic views of the AMMON core. The experimental zone loaded with 7 JHR-type fuel assemblies is surrounded by the driver zone (light grey). The water channels investigated by wire dosimetry are highlighted in white. (a) Measurements on the central assembly; (b) measurements on a peripheral assembly.

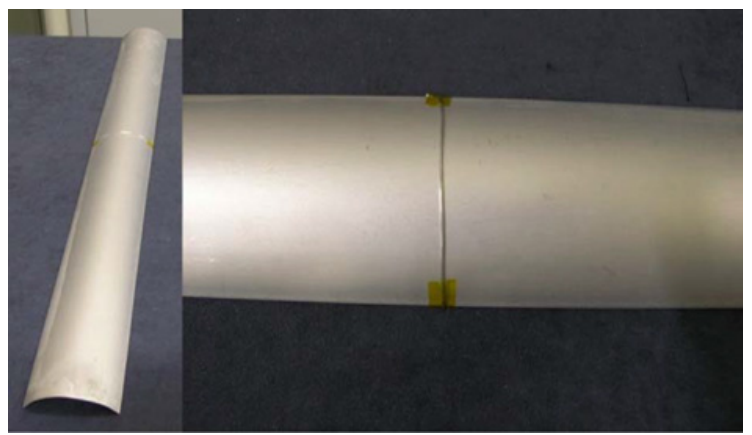

Figure 3. AMMON fuel plate with Al-Au wire for azimuthal capture rates measurements.

The reference wire segment (at the center of the plate) was measured up to 10 times on each detector to ensure the lowest achievable uncertainty. All other segments were measured once on each detector and normalized to the reference one. 


\section{Data Analysis}

\subsection{Net Area Determination}

To ensure good consistency of the analysis process with more than a thousand spectra, the NaI spectra were analyzed with an automated MATLAB routine to measure the count rate in the $411.8 \mathrm{keV}$ peak.

The peak shape is assumed to be the sum of a step-shaped background and a three-parameter Gaussian distribution:

$$
N_{t}(E)=a \mathrm{e}^{-\left(\frac{E-b}{c}\right)^{2}}+B
$$

where $N_{t}$ is the theoretical number of counts per channel $E ; a, b$, and $c$ are the fitted parameters; and $B$ is the background. The latter is evaluated upon 10 channels before and after the region of interest (ROI). The covariance matrix of the fitted parameters is computed from the Jacobian matrix $J_{p}$ of the parameters (the matrix of partial derivatives of the theoretical model with respect to the parameters) and from the diagonal matrix of the weights $w$ of the input data (the total counts per channel are considered independent):

$$
\operatorname{cov}(a, b, c)=\left(J_{p} \cdot w \cdot J_{p}^{T}\right)^{-1} .
$$

As the number of counts per channel follows a Poisson distribution, the uncertainty on the input data is assumed to follow the same law. The weights are then given by:

$$
w(E)=\frac{1}{N_{e}(E)}
$$

where $N_{e}(E)$ is the experimental number of counts per channel. Eventually, the variance of the peak area $A$ is given by:

$$
\operatorname{var}(A)=J_{A} \cdot \operatorname{cov}(a, b, c) \cdot J_{A}^{T}
$$

where $J_{A}$ is the Jacobian matrix of the partial derivatives of the peak area $A$ with respect to the fitted parameters $a, b$, and $c$.

The choice of the ROI in the spectrum is user-dependent, but remains the same for every spectrum acquired with each detector, in order to ensure consistency of the analysis.

\subsection{Normalized End-of-Irradiation Count Rates}

Overall measurements are combined as follows. For each wire on each detector, the end-of-irradiation count rate by mass unit $A_{m}$ (in counts.s. $\left.{ }^{-1} \mathrm{~g}^{-1}\right)$ is defined by:

$$
A_{m}=\frac{N}{m} \frac{\lambda}{\left(1-\mathrm{e}^{-\lambda t_{r}}\right) \mathrm{e}^{-\lambda t_{0}}}
$$

where $N$ is the count rate, $\lambda$ the gold decay constant (2.9775(9).10 $\left.10^{-6} \mathrm{~s}^{-1}[7]\right), t_{r}$ the real measurement time, $t_{0}$ the cooling time and $m$ the mass of the wire. For each wire measured once, uncertainty is calculated with Eq. (4). For the reference wire, measured $n$ times, the mean $A_{m, r e f}$ is given by:

$$
A_{m, r e f}=\frac{\sum_{i=1}^{n} A_{m, r e f, i} / u_{i}^{2}\left(A_{m, r e f}\right)}{\sum_{i=1}^{n} 1 / u_{i}^{2}\left(A_{m, r e f}\right)} .
$$




\section{$15^{\text {th }}$ ISRD}

Its uncertainty is given by:

$$
u^{2}\left(A_{m, r e f}\right)=\frac{1}{\sum_{i=1}^{n} 1 / u_{i}^{2}\left(A_{m, r e f}\right)}
$$

where $u_{i}^{2}\left(A_{m, r e f}\right)$ is equal to $\operatorname{var}\left(A_{m, r e f}\right)$, given by Eq. (4).

For each detector, each wire measurement is normalized to the reference and its uncertainty given by:

$$
A_{m, n o r m}=\frac{A_{m}}{A_{m, r e f}}, \quad \text { and } \quad u^{2}\left(A_{m, n o r m}\right)=u^{2}\left(A_{m}\right)+u^{2}\left(A_{m, r e f}\right) .
$$

Eventually, normalized measurements on the four detectors are combined to obtain the normalized end-of-irradiation count rates $A_{t o t}$ :

$$
\begin{gathered}
A_{\text {tot }}=\frac{\sum_{i=1}^{4} A_{m, \text { norm }, i} / u_{i}^{2}\left(A_{m, \text { norm }}\right)}{\sum_{i=1}^{4} 1 / u_{i}^{2}\left(A_{m, \text { norm }}\right)} \\
u^{2}\left(A_{\text {tot }}\right)=\frac{1}{\sum_{i=1}^{4} 1 / u_{i}^{2}\left(A_{m, n o r m}\right)}+u^{2}(m)+u^{2}\left(m_{r e f}\right) .
\end{gathered}
$$

The uncertainties on the masses are between $0.1 \%$ and $0.2 \%$. The overall final uncertainty lies below $1 \%$ (at 2 standard deviations). The uncertainty on the decay constant of gold has not been taken into account, as it is lower than $0.05 \%$.

\section{Results and Discussion}

Experimental results are presented below along with comparisons against calculations. The latter calculations were performed with the TRIPOLI4 Monte Carlo code [8], using a full three-dimensional representation of the AMMON core in the "hafnium" configuration [9].

For better understanding of the following graphs, all measurements were made clockwise with respect to Fig. 2.

\subsection{Central Assembly}

In this section, the gold capture rate distributions of plates 4 and 7 of the central assembly are presented.

On plate 4 of sector III, fission rate and capture rate calculations show that there are no perturbations linked to the nearby stiffener. Therefore, measurements on sector I and III are considered equivalent. The results presented here are the average of four irradiations ( 2 for sector I, 2 for sector III), representing 16 independent gamma ray measurements per wire part (each wire part is measured on four NaI detectors). Averaged normalized end-of-irradiation count rates uncertainties are lower than $0.5 \%$ on each point (at 2 standard deviations).

On the plate 4 edges, the gold capture rate shows an increase of about $4 \%$ compared to the central value (Fig. 4). Calculations and experiments show rather good agreement on the whole plate flux profile.

A comparison between calculated and measured capture rates profile for plate 7 of sector III is shown in Fig. 5. Experimental uncertainties lie around 1\% on each measurement point (at 2 standard deviations).

Calculation and experiment rates are in good agreement along the whole plate range. The gold capture increase at the plate edges is about $(4 \pm 1) \%$. However, the perturbation linked to the aluminum stiffener of the peripheral assembly, which should occur between 10 and $30 \mathrm{~mm}$, is not clear on the measured and calculated profiles. 


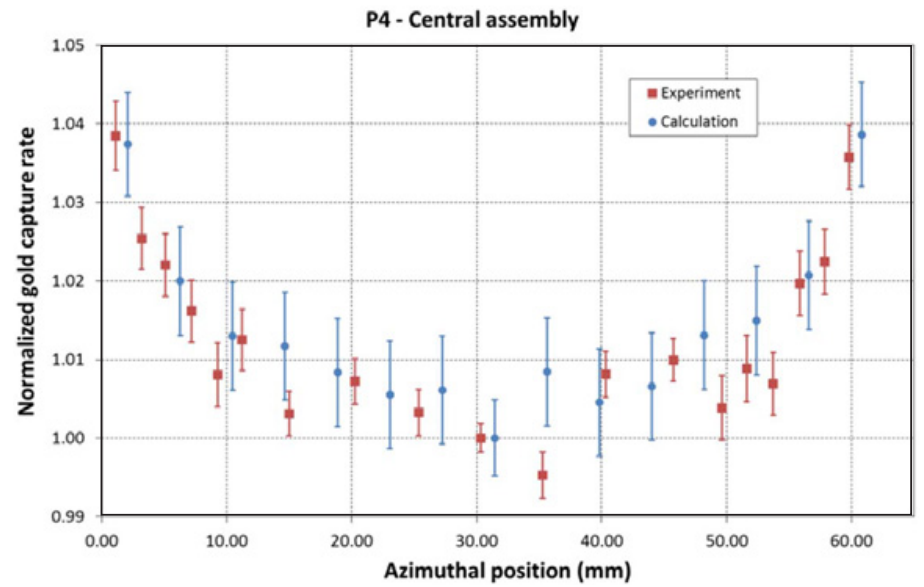

Figure 4. Experimental and calculated gold capture rate profiles in the water channels of plates 4 of the central assembly. Uncertainties are given at 2 standard deviations.

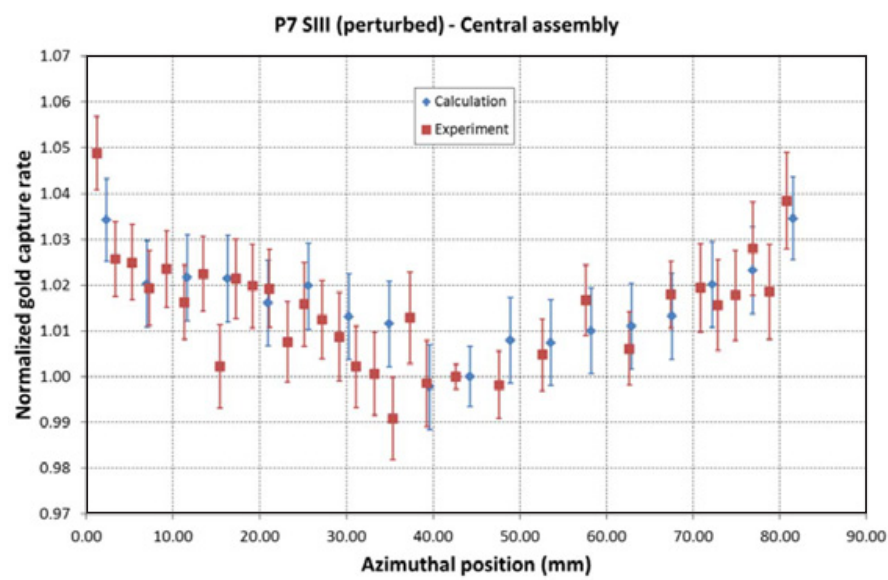

Figure 5. Experimental and calculated gold capture rate profiles in the water channel of plate 7 of sector III of the central assembly, perturbed by the stiffener of the peripheral assembly. Uncertainties are given at 2 standard deviations.

\subsection{Peripheral Assembly}

A comparison between calculated and measured capture rate profiles is shown on Fig. 6, for the plate 7 of the peripheral assembly, in front of the central one (see Fig. 2(b)).

The influence of the assembly position (at the periphery) is clearly noticeable. It leads to a decrease of about $4 \%$ of the capture rate on the left-hand side of the graph (corresponding to the outer edge), compared to the center. On the right hand side of the graph (corresponding to the side of the plate in front of the central assembly), the flux increase is similar to the unperturbed configuration (5\% to $6 \%$ compared to the center). Along the whole plate width, the thermal and epithermal flux decrease from the inner to the outer edge is around $10 \%$.

Calculations and experiments rates are in close agreement along the whole plate width, except for the points the furthest to the right-hand side (the inner edge of the plate, close to the central assembly), which show a $4 \%$ discrepancy, inconsistent with their uncertainties. This variation may be linked to 


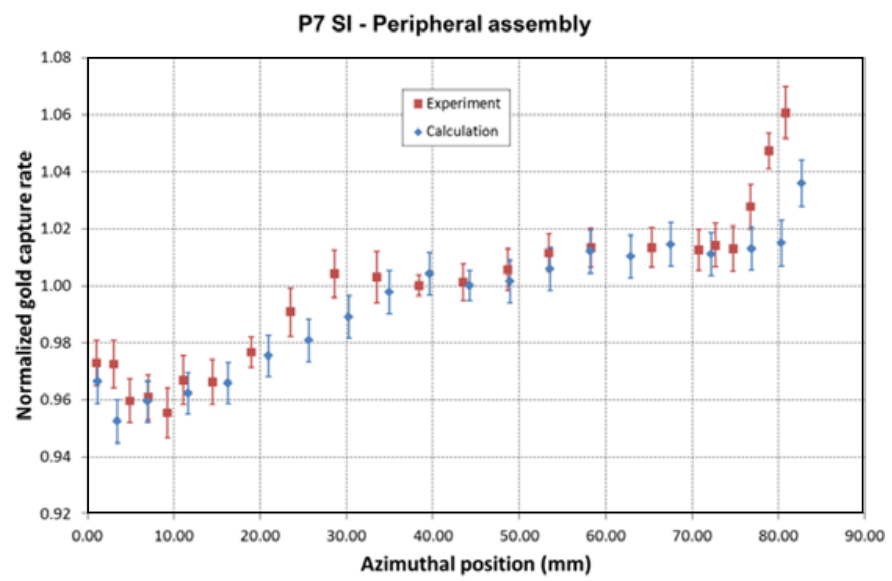

Figure 6. Experimental and calculated gold capture rate profiles in the water channel of the sector I plate 7 of the peripheral assembly. Uncertainties are given at 2 standard deviations.

technological uncertainties on the precise shape of this plate of the dismountable assembly and to a local dog-bone effect on the edges of the fuel material. These uncertainties or dog-bone effect can have an impact on the water blade thickness or on the neutron energy distribution around the fuel elements, on the capture rate profile.

\section{Conclusions}

Gold wire dosimetry measurements were carried out during the AMMON experimental program, in the "hafnium" configuration, to assess the thermal and epithermal flux distribution between fuel plates. Besides flux shape in water channels, two other effects were investigated: the flux distortion linked to the aluminum stiffener of a nearby assembly and the flux distortion linked to the assembly position in the AMMON core. Moreover, experimental results were compared to full 3D Monte Carlo calculations.

The overall uncertainty of the normalized activity of each wire lies below $1 \%$ (at 2 standard deviations).

The position of a peripheral assembly in the core leads to a decrease of about $10 \%$ of the gold capture rate between the two sides of the outer plate. Except for points on the plates' inner edge, calculation and experiment are in close agreement. For measurements on plates 4 and 7 of the central assembly, the gold capture rate increase on their edges is also well described.

No clear impact on the thermal and epithermal flux linked to a nearby assembly stiffener was observed by calculation or experiment.

\section{References}

[1] J.-C. Klein et al., AMMON: an experimental program in the EOLE critical facility for the validation of the Jules Horowitz Reactor neutron and photon HORUS3D calculation scheme. Proc. Int. Conf. IGORR 2009, Beijing, China (2009)

[2] P. Fougeras, A. Chabre, C. Mergui, The place of EOLE, MINERVE and MASURCA facilities in the R\&D activities of the CEA, Proc. Int. Conf. IGORR 2005, Gaitherburg, USA (2005)

[3] D. Iracane, The Jules Horowitz Reactor, a new Material Testing Reactor in Europe. Proc. Int. Conf. IGORR 2005, Gaitherburg, USA (2005) 
[4] A. Gruel et al., Design of a devoted gamma spectrometry device for axial and azimuthal activity measurements on JHR-type curved fuel plates, IEEE Trans. Nucl. Sci. 61 (2014)

[5] C. Vaglio-Gaudard et al., Monte Carlo Interpretation of the AMMON/REF Experiment in EOLE for the JHR Reactor Neutron Calculations, Nucl. Sci. Eng. 175 (2013)

[6] J.-M. Girard, H. Philibert, S. Testanière, C. Domergue, D. Beretz, The MADERE radio-activity measurement Platform: developments for a better address of the experimental needs. Proc. Int. Conf. IGORR 2009, Beijing, China (2009)

[7] LNHB, PTB/E. Schönfeld, R. Dersch (1998), http: //laraweb.free.fr/

[8] E. Brun et al., Overview of TRIPOLI-4@ version 7 continuous-energy Monte Carlo transport code. Proc. Int. Conf. ICAPP 2011, Nice, France, May (2011)

[9] C. Vaglio-Gaudard et al., Analysis of the AMMON experimental program in the EOLE facility supporting the qualification of the JHR neutron and photon tools, IEEE Trans. Nucl. Sci. 61 (2014) 